\title{
REGULARITY RESULTS FOR AN ELLIPTIC-PARABOLIC FREE BOUNDARY PROBLEM
}

\author{
M. BERTSCH AND J. HULSHOF
}

\begin{abstract}
We study an elliptic-parabolic free boundary problem in one space dimension. We give several regularity results for both the weak solution and the free boundary. In particular conditions are given which ensure that the free boundary is a $C^{1}$-curve.
\end{abstract}

1. Introduction. Consider the initial-boundary value Problem I:

$$
\begin{cases}(c(u))_{t}=u_{x x} & \text { in } Q_{T}=(0,1) \times(0, T] \\ u_{x}(0, t)=0 \text { and } u_{x}(1, t)=f(t), & 0<t \leq T, \\ c(u(x, 0))=v_{0}(x), & 0 \leq x \leq 1\end{cases}
$$

Here $T>0$ and the functions $c, f$ and $v_{0}$ satisfy the following hypotheses.

H1. $c \in C(\mathbf{R}) \cap C^{2, \alpha}\left(\mathbf{R}^{-}\right)$for some $\alpha \in(0,1), c \equiv 1$ on $\mathbf{R}^{+}$, and $c^{\prime}$ is strictly positive and uniformly bounded on $\mathbf{R}^{-}$.

H2. $f:[0, T] \rightarrow \mathbf{R}$ is Lipschitz continuous and strictly positive.

H3. There exists a Lipschitz continuous function $u_{0}:[0,1] \rightarrow \mathbf{R}$ such that $v_{0}=$ $c\left(u_{0}\right)$.

H4. $\int_{0}^{1} v_{0}(x) d x+\int_{0}^{T} f(t) d t<1$.

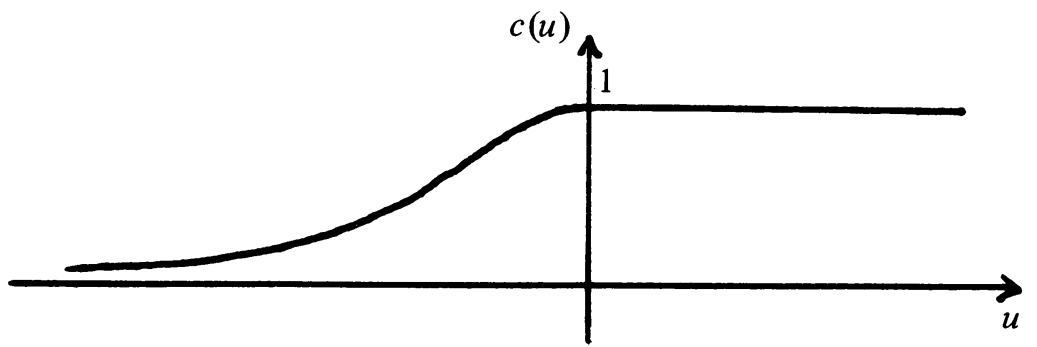

FIGURE 1. The function $c(u)$

Problem I describes the one dimensional fluid flow in a partially saturated porous medium: $u$ denotes the hydrostatic potential due to capillary suction, $c$ the moisture content, and we assume that $c$ depends on $u$ as described in Figure 1. The saturation value of $c$ is taken to be one:

$$
c(u(x, t))=1 \Leftrightarrow u(x, t) \geq 0 \Leftrightarrow \text { saturation at the point }(x, t) .
$$

From a mathematical point of view equation (1.1) is quite interesting. It is of parabolic type at points $(x, t)$ where the medium is unsaturated, i.e., where

Received by the editors September 12, 1985.

1980 Mathematics Subject Classification. Primary 35B99. 
$u(x, t)<0\left(\Leftrightarrow c^{\prime}(u)>0\right)$, and of elliptic type in the saturated region where $u(x, t) \geq 0\left(\Leftrightarrow c^{\prime}(u)=0\right)$. In particular the answer to the question whether Problem I has a classical solution is not a priori clear.

Problem I does have a unique weak solution $[6,8]$. By this statement we mean that there exists a unique function $u \in L^{2}\left(0, T ; H^{1}(0,1)\right)$ which satisfies

(i) $c(u) \in C\left(\bar{Q}_{T}\right)$;

(ii) for every test function $\phi \in C^{1}\left(\bar{Q}_{T}\right)$ vanishing at $t=T$

$$
\iint_{Q_{T}}\left\{\phi_{x} u_{x}-\phi_{t} c(u)\right\} d x d t=\int_{0}^{1} \phi(x, 0) v_{0}(x) d x+\int_{0}^{T} \phi(1, t) f(t) d t
$$

(for more general existence results we refer to $[\mathbf{1}]$ ). We observe here that condition $\mathrm{H} 4$ is natural in view of the conservation law

$$
\int_{0}^{1} c(u(x, t)) d x=\int_{0}^{1} v_{0}(x) d x+\int_{0}^{t} f(s) d s, \quad 0 \leq t \leq T,
$$

and it expresses the fact that the medium is not completely saturated at time $T$.

Of special interest is the free boundary between the regions where the medium is saturated, respectively, unsaturated. We define the set

$$
P_{t}=\{0 \leq x \leq 1: c(u(\cdot, t)) \equiv 1 \text { on }[x, 1]\}
$$

and the function $\zeta:[0, T] \rightarrow[0,1]$ by

$$
\zeta(t)= \begin{cases}\inf P_{t} & \text { if } P_{t} \neq \varnothing \\ 1 & \text { if } P_{t}=\varnothing\end{cases}
$$

Hulshof $[6]$ has shown that

$$
\zeta \text { is continuous on }[0, T]
$$

and, if $0<\varsigma(t)<1$, the point $x=\varsigma(t)$ is the interface between the saturated and the unsaturated region:

$$
\begin{array}{lll}
c(u(x, t))<1 & \text { if } 0 \leq x<\varsigma(t), & 0<t \leq T \\
c(u(x, t))=1 & \text { if } \varsigma(t)<x \leq 1, & 0 \leq t \leq T .
\end{array}
$$

Since $c(u(\cdot, t)) \not \equiv 1$ on $[0,1]$ it follows that $\zeta>0$ on $[0, T]$. It may happen however that, for some $t \in(0, T], \zeta(t)=1$. In that case it is possible that $c(u(\zeta(t), t))<1$, i.e. the medium is completely unsaturated at that time.

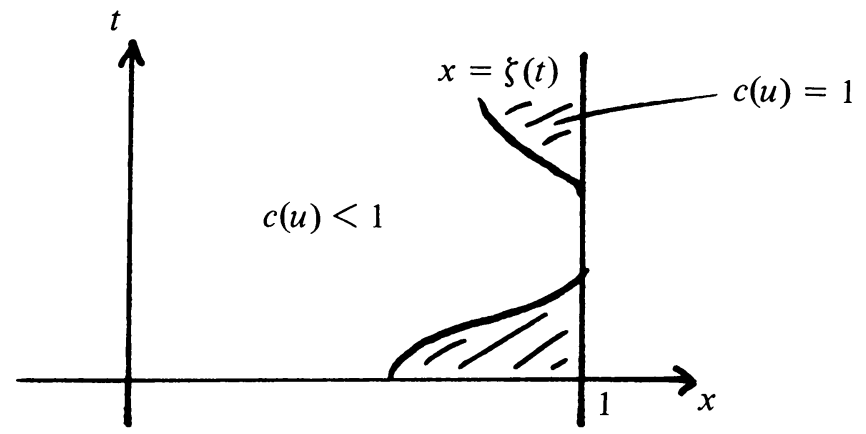

FIGURE 2 
In this paper we present some regularity results for the function $\zeta$ which improve (1.5). We shall give conditions which guarantee that $\zeta$ is Lipschitz continuous, respectively continuously differentiable. We also prove regularity results for the weak solution $u$ of Problem I. In particular we shall give conditions such that $u$ is a classical solution.

Before we state the precise results, we give a list of several additional assumptions on the data.

A1. $c(s)$ is concave near $s=0$.

A2. $u_{0} \in C^{2, \alpha}([a, \zeta(0))) \cap C^{1}([a, \zeta(0)])$, where $\alpha \in(0,1), \varsigma(0)$ is defined by $(1.4)$ and $a \in[0, \varsigma(0))$;

$$
\left|u_{0}^{\prime \prime}\right| \leq M c^{\prime}\left(u_{0}\right) \quad \text { on }[a, \varsigma(0)) \quad(M>0),
$$

$u_{0}$ is piecewise monotone, and $u_{0}^{\prime}(\varsigma(0))=f(0)$.

$\mathrm{A2}^{*}$. $u_{0}$ satisfies $\mathrm{A} 2, u_{0} \in C^{3, \alpha}([a, \varsigma(0)))$ and $u_{0}^{\prime \prime} / c^{\prime}\left(u_{0}\right)$ is uniformly Lipschitz continuous on $[a, \zeta(0))$.

Without loss of generality we may assume that $u_{0}<0$ on $[a, \zeta(0))$.

THEOREM 1.1. Let the hypotheses $\mathrm{H} 1-\mathrm{H} 4$ and $\mathrm{A} 2$ be satisfied and let $u$ be the weak solution of Problem I. If either $c(u(1, \cdot)) \equiv 1$ on $[0, T]$ or c satisfies assumption A1, then

(i) $\zeta$ is Lipschitz continuous on $[0, T]$;

(ii) $u_{x} \in C([0,1] \times(0, T])$ and

$$
u_{x}(x, t)=f(t) \quad \text { if } \varsigma(t) \leq x \leq 1,0 \leq t \leq T
$$

(iii) $u_{t} \in L_{\mathrm{loc}}^{\infty}([0,1] \times(0, T])$;

(iv) if $c^{\prime}\left(0^{-}\right)=0$ then $u$ is a classical solution of Problem I, i.e. $(c(u))_{t} \in$ $C\left(Q_{T}\right), u_{x x} \in C\left(Q_{T}\right)$ and $u$ satisfies (1.1)-(1.3) pointwise.

If we replace condition $\mathrm{A} 2$ by the stronger condition $\mathrm{A} 2{ }^{*}$ we can improve Theorem 1.1.

THEOREM 1.2. Let the hypotheses $\mathrm{H} 1-\mathrm{H} 4$ and $\mathrm{A}^{*}$ be satisfied and let $u$ be the weak solution of Problem I. If either $c(u(1, \cdot)) \equiv 1$ on $[0, T]$, or $c$ satisfies assumption A1, then

(i) $\zeta$ is continuously differentiable in every $t \in[0, T]$ where $\zeta(t)<1$;

(ii) $u_{x t} \in L_{\text {loc }}^{\infty}([0,1] \times(0, T])$;

(iii) $u_{t} \in C\left(\bar{D} \cap Q_{T}\right)$, where

$$
D=\left\{(x, t) \in Q_{T}: 0<x<\zeta(t)\right\},
$$

and if $\varsigma(t)<1$

$$
\zeta^{\prime}(t)=-u_{t}\left(\zeta(t)^{-}, t\right) / f(t)
$$

(iv) if $f \in C^{1}([0, T])$ then $u_{t} \in C\left(Q_{T}\right)$.

REMARK 1 . In Theorems 1.1 and 1.2 the set $[0,1] \times(0, T]$ may be replaced by any compact subset $K$ of $\bar{Q}_{T}$ which does not contain the set $\{(x, t): 0 \leq x \leq a, t=0\}$.

REMARK 2. If $c^{\prime}\left(0^{-}\right)>0$ the weak solution is in general not classical in the neighborhood of a point $\left(\zeta\left(t_{0}\right), t_{0}\right)$ with $\zeta\left(t_{0}\right)<1$. Otherwise (1.8) and the fact that $(c(u))_{t}=u_{x x}=0$ in $\left\{(x, t) \in Q_{T}: \zeta(t)<x<1\right\}$ would imply

$$
\varsigma^{\prime}\left(t_{0}\right)=\frac{-u_{t}\left(\varsigma\left(t_{0}\right)^{-}, t_{0}\right)}{f\left(t_{0}\right)}=\frac{-\left(c\left(u\left(\varsigma\left(t_{0}\right), t_{0}\right)\right)\right)_{t}}{c^{\prime}\left(u\left(\varsigma\left(t_{0}\right)^{-}, t_{0}\right)\right) f\left(t_{0}\right)}=0 .
$$


REMARK 3. The condition $\zeta(t)<1$ in Theorem $1.2(\mathrm{i})$, (iii) cannot be omitted. It can be shown that in general $\zeta$ arrives at, respectively departs from the lateral boundary $\{x=1\}$ with nonzero speed.

REMARK 4. The condition that $f \in C^{1}([0, T])$ in Theorem $1.2(\mathrm{iv})$ cannot be omitted. This follows at once from the observation that, since $u_{x x}=0$ in $Q_{T} \backslash D, u$ is given by

$$
u(x, t)=f(t)\{x-\varsigma(t)\} \quad \text { if } \varsigma(t)<x<1 .
$$

REMARK 5 . We believe that the conditions A2 resp. A2* are not necessary for the results of Theorem 1.1 (resp. Theorem 1.2). The proofs of these theorems are based on bounds for the functions $u_{t}$ and $u_{x t}$ near the interface $x=\zeta(t)$. To obtain these bounds, we need that $u_{t}$ (resp. $u_{x t}$ ) are bounded at $t=0$ near $x=\varsigma(0)$. Since formally, from equation (1.1), $u_{t}=u_{x x} / c^{\prime}(u)$, we arrive at the conditions on $u_{0}^{\prime \prime} / c^{\prime}\left(u_{0}\right)$ in the assumptions $\mathrm{A} 2$ and $\mathrm{A} 2^{*}$. We expect however that equation (1.1) has a regularizing effect for $t>0$, i.e. also if $\mathrm{A} 2$ and $\mathrm{A} 2^{*}$ do not hold, the function $u_{x x} / c^{\prime}(u)$ and its spatial derivative are bounded for every $t>0$. In that case the theorems would still be valid (except for the smoothness of $\zeta$ at $t=0$ ).

REMARK 6. Our method of proof is based on the maximum principle, i.e. we use global techniques which depend heavily on the boundary conditions. Not only the method, but also the results depend on the boundary condition at $x=1$. It follows for example from results in [7] that, if we drop our condition $f>0$ on $[0, T]$, it is possible to construct a solution with a discontinuous function $\zeta$. So we cannot expect that all our results can be proved with local techniques.

In this connection we mention that DiBenedetto and Gariepy [3] use local techniques to prove that, in arbitrary space dimensions, for bounded weak solutions, $c(u)$ is continuous.

The proofs of Theorem 1.1 and 1.2 are given in $\S 4$ resp. $\S 5$ of this paper. $\S 3$ is devoted to the study of the level lines of $u$ near the interface curve $x=\varsigma(t)$ (observe that, at least formally, $x=\varsigma(t)$ itself is a level line, namely $\{u=0\}$, provided $\zeta(t)<1$ ). In $\S 6$ we comment on generalizations of our results to the case of a Dirichlet boundary condition at $x=1$.

2. Preliminaries. First we collect some known results about the solution of Problem I [6, 8].

Proposition 2.1. Let $\mathrm{H} 1-\mathrm{H} 4$ be satisfied. Then Problem I has a unique weak solution $u$, which has the following properties.

(i) $u \in L^{2}\left(0, T ; H^{2}(0,1)\right) \cap L^{\infty}\left(0, T ; W^{1, \infty}(0,1)\right)$.

(ii) $c(u) \in C^{0+1}\left(\bar{Q}_{T}\right) \cap H^{1}\left(Q_{T}\right)$.

(iii) $u$ is a classical solution in the region

$$
D=\left\{(x, t) \in Q_{T}: c(u(x, t))<1\right\} .
$$

(iv) The equalities in Problem I all hold in the a.e. sense.

(v) The function $\zeta$, defined by (1.4) satisfies (1.6) and $\zeta \in C([0, T])$.

By Proposition 2.1(iv) $u_{x x}=0$ a.e. in $Q_{T} \backslash D$ and, by Proposition 2.1(i), $u(\cdot, t) \in$ $C^{1}([0,1])$ for a.e. $t \in[0, T]$. Since $u_{x}(1, t)=f(t)$ a.e. on $[0, T]$, this implies that we may assume, after redefining $u$ on a set of measure zero, that

$$
u(x, t)=f(t)\{x-\varsigma(t)\} \quad \text { on } \overline{Q_{T} \backslash D} .
$$


The existence of a weak solution was proved in [8] by means of parabolic regularization. In this method the functions $c, u_{0}$ and $f$ are replaced by smooth functions $c_{n}, u_{0 n}$ and $f_{n}$ with the properties

(i) $c_{n} \rightarrow c ; c_{n}\left(u_{0 n}\right) \rightarrow v_{0}$, and $f_{n} \rightarrow f$ as $n \rightarrow \infty$;

(ii) $\left|u_{0 n}^{\prime}\right| \leq L, u_{0 n}^{\prime}(0)=0$, and $u_{0 n}^{\prime}(1)=f_{n}(0)$;

(iii) $1 / n \leq c_{n}^{\prime} \leq K<\infty$.

Let $u_{n}(x, t)$ denote the unique solution of Problem $\mathrm{I}_{n}$ :

$$
\begin{cases}\left(c_{n}(u)\right)_{t}=u_{x x} & \text { in } Q_{T} \\ u_{x}(0, t)=0 \text { and } u_{x}(1, t)=f_{n}(t), & 0<t \leq T \\ u(x, 0)=u_{0 n}(x), & 0 \leq x \leq 1\end{cases}
$$

Then $u_{n} \rightarrow u$ as $n \rightarrow \infty$.

The proofs of Theorems 1.1 and 1.2 are based on estimates of $u_{n}$ which are uniform with respect to $n$. For that reason we have to reconstruct the initial functions $u_{0 n}$ in such a way that they satisfy the conditions A2 and A2* , uniformly with respect to $n$.

LEMMA 2.2. Let $u_{0}$ satisfy assumption A2. Then there exists a sequence of smooth functions $u_{0 n}:[0,1] \rightarrow \mathbf{R}$ such that

$$
\begin{array}{ll}
c_{n}\left(u_{0 n}\right) \rightarrow v_{0} & \text { in } C([0,1]) \text { as } n \rightarrow \infty, \\
u_{0 n} \rightarrow u_{0} & \text { in } C^{1}([a, \zeta(0)]) \text { as } n \rightarrow \infty, \\
u_{0 n}^{\prime} \rightarrow f(0) & \text { in } C([\zeta(0), 1]) \text { as } n \rightarrow \infty, \\
u_{0 n}^{\prime}(0)=0, &
\end{array}
$$

and

$$
\left|u_{0 n}^{\prime \prime}\right| \leq M c_{n}^{\prime}\left(u_{0 n}\right) \quad \text { on }[a, 1] .
$$

If $u_{0}$ satisfies $\mathrm{A} 2^{*}$, we have in addition that

$$
u_{0 n}^{\prime \prime} / c_{n}^{\prime}\left(u_{0 n}\right) \text { is uniformly bounded in } C^{1}([a, 1]) \text {. }
$$

REMARK. Once we have constructed the functions $u_{0 n}$, we can choose the functions $f_{n}$ in Problem $\mathrm{I}_{n}$ such that they satisfy the compatibility condition $u_{0 n}^{\prime}(1)=f_{n}(0)$.

PROOF. There exists a sequence of functions $q_{n} \in C^{\infty}([a, 1])$ such that

$$
\left|q_{n}\right| \leq M \quad \text { on }[a, 1]
$$

and

$$
q_{n} \rightarrow u_{0}^{\prime \prime} / c^{\prime}\left(u_{0}\right) \quad \text { in } C([a, \varsigma(0)-\varepsilon)) \text { as } n \rightarrow \infty
$$

for any small $\varepsilon>0$.

Let $u_{0 n}:[a, 1] \rightarrow \mathbf{R}$ be the unique solution of the initial-value problem

$$
\left\{\begin{array}{l}
w^{\prime \prime}=q_{n} c_{n}^{\prime}(w), \quad a<x \leq 1, \\
w(a)=u_{0}(a) \text { and } w^{\prime}(a)=u_{0}^{\prime}(a) .
\end{array}\right.
$$

Here $c_{n}$ is a sequence of smooth functions such that

$1 / n \leq c_{n}^{\prime} \leq K$ on $\mathbf{R}$,

$c_{n}^{\prime} \rightarrow 0$ uniformly on $[\delta, \infty)$ for all $\delta>0$, 
$c_{n} \rightarrow c$ in $C^{2}(I)$ for every compact set $I \subset \mathbf{R}^{-}$.

We extend $u_{0 n}$ to a smooth function on $[0,1]$ in such a way that $u_{0 n}^{\prime}(0)=0,\left|u_{0 n}^{\prime}\right| \leq$ $L$, and $c_{n}\left(u_{0 n}\right) \rightarrow v_{0}$ on $[0, a]$.

We claim that $u_{0 n}$ satisfies (2.2)-(2.4).

To prove (2.2), it is sufficient to show that $u_{0 n} \rightarrow u_{0}$ in $C^{1}([a, \zeta(0)])$ as $n \rightarrow \infty$. Since $u_{0 n}$ is uniformly bounded in $C^{2}([a, \zeta(0)])$, there exists a subsequence (which we denote by $\left\{u_{0 n}\right\}$ again) such that $u_{0 n} \rightarrow w$ in $C^{1}([a, \zeta(0)])$ as $n \rightarrow \infty$ for some $w \in C^{1}([a, \varsigma(0)])$. We have to show that

$$
w=u_{0} \quad \text { on }[a, \zeta(0)] .
$$

From the construction of $q_{n}, c_{n}$, and $u_{0 n}$, it can be easily derived that the limit function $w$ is the unique solution of the initial value problem

$$
\begin{cases}w^{\prime \prime}=u_{0}^{\prime \prime} c^{\prime}(w) / c^{\prime}\left(u_{0}\right), & a<x \leq \varsigma(0)-\varepsilon \\ w(a)=u_{0}(a), & w^{\prime}(a)=u_{0}^{\prime}(a)\end{cases}
$$

where $\varepsilon>0$ is an arbitrarily small number. Thus, by uniqueness, $w=u_{0}$ on $[a, \zeta(0)-\varepsilon]$ and $(2.8)$ follows.

From (2.6) and the construction of $u_{0 n}$ we find at once that (2.4) is satisfied.

To prove (2.3) we observe that, by $(2.2)$,

$$
u_{0 n}^{\prime}(\varsigma(0)) \rightarrow f(0) \quad \text { as } n \rightarrow \infty,
$$

since $u_{0}^{\prime}(\zeta(0))=f(0)$. We know that $f(0)>0, u_{0 n}(\zeta(0)) \rightarrow 0$ as $n \rightarrow \infty, u_{0}^{\prime \prime}$ is uniformly bounded, and that $c_{n}^{\prime} \rightarrow 0$ uniformly on $[\delta, \infty)$ for all $\delta>0$. Combining these facts we obtain (2.3).

Finally, if $u_{0}$ satisfies $\mathrm{A} 2^{*}$, we can choose the functions $q_{n}$ such that

$$
q_{n}^{\prime} \text { is uniformly bounded on }[a, 1]
$$

and

$$
q_{n} \rightarrow q_{\infty} \text { in } C([a, 1]),
$$

where $q_{\infty} \in C([a, 1])$ is defined by

$$
q_{\infty}(x)= \begin{cases}\frac{u_{0}^{\prime \prime}(x)}{c^{\prime}\left(u_{0}(x)\right)}, & a \leq x<\varsigma(0), \\ \lim _{x \uparrow \varsigma(0)} \frac{u_{0}^{\prime \prime}(x)}{c^{\prime}\left(u_{0}(x)\right)}, & \varsigma(0) \leq x<1,\end{cases}
$$

and (2.5) is clearly satisfied.

3. The level curves. In the Introduction we mentioned already that, as long as $\zeta(t)<1$, the set $x=\zeta(t)$ is, at least formally, the level curve $\{(x, t): u(x, t)=0\}$. Since our main objective is to study the regularity of $\zeta$, it seems a natural approach to study the level curves of $u$ in a neighborhood of $x=\varsigma(t)$ (see also [2]).

Our plan is as follows. First, to eliminate the formal aspect of calculations, we shall study the level curves of the approximating smooth solutions $u_{n}$ of Problem $\mathrm{I}_{n}$ which we introduced in $\S 2$. Given a level curve $x=X(t)$ of $u_{n}$, it follows from $(d / d t)\left\{u_{n}(X(t), t)\right\}=0$ that

$$
X_{t}=-u_{n t} / u_{n x} \quad \text { if } u_{n x} \neq 0
$$


Defining

$$
\eta_{n}(x, t)=-u_{n t}(x, t) / u_{n x}(x, t) \quad \text { if } u_{n x}(x, t) \neq 0
$$

we derive a parabolic partial differential equation for $\eta_{n}$. In the following sections this equation will be the main tool to obtain uniform bounds for $\eta_{n}$, which, by (3.1), are bounds for the time derivatives of level curves.

Secondly, to control the condition $u_{n x} \neq 0$ in $(3.1)$, we shall prove that the condition $f(t)>0$ in (1.2) implies that $u_{n x} \geq \delta>0$ in a neighborhood of $x=\varsigma(t)$ for $n$ large enough and for some $\delta>0$.

LEMMA 3.1. Let $u_{n}$ be the solution of Problem $\mathrm{I}_{n}$. If $u_{n x} \neq 0$ at $\left(x_{0}, t_{0}\right) \in Q_{T}$ then the function $\eta_{n}$, defined by (3.2), satisfies

$$
\eta_{t}=\eta_{x x} / c_{n}^{\prime}\left(u_{n}\right)-\left(\eta^{2}\right)_{x} \text { at }\left(x_{0}, t_{0}\right) \text {. }
$$

Proof. Although it is possible to obtain (3.3) by direct calculation, we choose a proof which uses the level curves of $u_{n}$ as a new coordinate system, following an idea by Gurtin, MacCamy, and Socolovsky [5].

For convenience we drop the subscript $n$.

Since $u_{x} \neq 0$ in a neighborhood $\mathcal{V}$ of $\left(x_{0}, t_{0}\right)$, we can define in $\mathcal{V}$ a coordinate transformation $(x, t) \rightarrow(p, \tau)$ defined by $x=X(p, \tau), t=\tau$, where $X$ is defined by $u(X(p, \tau), \tau)=p$. Hence $u_{t}+u_{x} X_{\tau}=0$ and $u_{x} X_{p}=1$. Using this we derive that

$$
\frac{1}{X_{p}} X_{\tau}=u_{x} X_{\tau}=-u_{t}=-\frac{u_{x x}}{c^{\prime}(u)}=-\frac{1}{c^{\prime}(u)}\left(\frac{1}{X_{p}}\right)_{p} \frac{1}{X_{p}}=\frac{1}{c^{\prime}(u)} \frac{X_{p p}}{X_{p}^{3}}
$$

or

$$
X_{\tau}=X_{p p} / c^{\prime}(p) X_{p}^{2}
$$

We set $Y(p, \tau)=X_{\tau}(p, \tau)$ and differentiate (3.4) with respect to $\tau$. This yields

$$
Y_{\tau}=\frac{1}{c^{\prime}(p)} \frac{Y_{p p}}{X_{p}^{2}}-\frac{2 X_{p p}}{c^{\prime}(p) X_{p}^{3}} Y_{p}=\frac{1}{c^{\prime}(p)} \frac{Y_{p p}}{X_{p}^{2}}-\frac{2 Y Y_{p}}{X_{p}}
$$

where we used (3.4) another time. Defining

$$
\eta(x, t)=Y(p, \tau)
$$

we find that

$$
\eta_{t}=Y_{\tau}-\eta_{x} \eta=\frac{1}{c^{\prime}(u)} \eta_{x x}+\frac{1}{c^{\prime}(u)} \frac{X_{p p}}{X_{p}^{2}} \eta_{x}-2 \eta \eta_{x}-\eta \eta_{x}=\frac{1}{c^{\prime}(u)} \eta_{x x}-2 \eta \eta_{x}
$$

which completes the proof.

In the following lemmas we establish lower bounds for $u_{x}$ and $u_{n x}$ near $x=\varsigma(t)$.

LEMMA 3.2. Let hypotheses $\mathrm{H} 1-\mathrm{H} 4$ be satisfied and let $u_{0}$ be piecewise monotone. Let $u$ be the solution of Problem I. For every $t_{0} \in[0, T]$ there exists a neighborhood $N\left(t_{0}\right)$ of $\left(\varsigma\left(t_{0}\right), t_{0}\right)$ in $\overline{Q_{T}}$ such that $u_{x} \geq 0$ a.e. in $N\left(t_{0}\right)$.

PROOF. If $\varsigma\left(t_{0}\right)=1$ and $c\left(u\left(\varsigma\left(t_{0}\right), t_{0}\right)\right)<1, u$ is a classical solution of Problem I in a neighborhood of $\left(\zeta\left(t_{0}\right), t_{0}\right)$. Since $u_{x}\left(\zeta\left(t_{0}\right), t_{0}\right)=f\left(t_{0}\right)>0$, the existence of $N\left(t_{0}\right)$ is obvious.

So let $c\left(u\left(\varsigma\left(t_{0}\right), t_{0}\right)\right)=1$. Since $u_{0}$ is piecewise monotone, it follows from the socalled lap-number arguments in $[6$, Appendix $]$ that the function $u(\cdot, t)$ is piecewise 
monotone on $[0, \zeta(t)]$ for all $t \in[0, T]$. Thus there exists for every $t$ a number $\phi(t) \in[0, \zeta(t))$ such that $u_{x}(\cdot, t) \geq 0$ on $[\phi(t), \zeta(t))$. Here we recall that $u$ is a classical solution in $D$ and hence $u_{x}$ is a smooth function. We may choose $\phi(t)$ as small as possible. Observe that $u_{x}(\cdot, t) \not \equiv 0$ on $[\phi(t), \zeta(t)]$.

We now argue by contradiction. Since by (2.1) $u_{x}=f>0$ if $x>\varsigma(t)$, we suppose that there exists a sequence $\left(x_{n}, t_{n}\right) \in D$ with $\left(x_{n}, t_{n}\right) \rightarrow\left(\varsigma\left(t_{0}\right), t_{0}\right)$ as $n \rightarrow \infty$ and $u_{x}\left(x_{n}, t_{n}\right)<0$.

First we consider the case that $t_{n} \nearrow t_{0}$ as $n \rightarrow \infty$. For all $n$ there exists a maximal interval $\left(y_{n}, z_{n}\right)$ with $0 \leq y_{n}<x_{n}<z_{n}<\varsigma\left(t_{n}\right)$, such that $u_{x}\left(\cdot, t_{n}\right) \leq 0$ on $\left(y_{n}, z_{n}\right)$. Hence $c\left(u\left(\cdot, t_{n}\right)\right)$ attains a local maximum in $y_{n}$. Since $u\left(y_{n}, t_{n}\right) \geq$ $u\left(x_{n}, t_{n}\right)$ and since, by the continuity of $\zeta, c\left(u\left(x_{n}, t_{n}\right)\right) \rightarrow 1$ as $n \rightarrow \infty$, we have that

$$
c\left(u\left(y_{n}, t_{n}\right)\right) \rightarrow 1 \text { as } n \rightarrow \infty .
$$

Since $u(\cdot, t)$ is piecewise monotone, the largest local maximum of $c(u(\cdot, t))$ less than one, which we denote by $S(t)$, is well defined. In [6] it was proved that $S(t)$ is decreasing with respect to $t$. Hence

$$
S\left(t_{n}\right) \nrightarrow 1 \text { as } n \rightarrow \infty,
$$

which is a contradiction to (3.5).

Finally we consider the case that $t_{n} \searrow t_{0}$ as $n \rightarrow \infty$. We choose $\bar{x} \in\left(\phi\left(t_{0}\right), \varsigma\left(t_{0}\right)\right)$ and $\tau>t_{0}$ such that $u_{x}(\bar{x}, \cdot)>0$ on $\left[t_{0}, \tau\right]$. Using that, by (2.1), $u_{x}=f$ in $Q_{T} \backslash \bar{D}$, it follows that $u_{x} \geq 0$ on the parabolic boundary of the set $(\bar{x}, 1) \times\left(t_{0}, \tau\right]$. By the maximum principle for $u_{x}$ (which can be easily proved using the approximating sequence $\left.\left\{u_{n}\right\}\right)$ it follows that $u_{x} \geq 0$ a.e. in this set, and we obtain a contradiction.

LEMMA 3.3. Let the assumptions of Lemma 3.2 be satisfied and let $u_{0} \in$ $C^{1}([a, 1])(0 \leq a<\varsigma(0))$ with $u_{0}^{\prime}(\varsigma(0))=f(0)$. Then there exist a function $\xi \in C^{\infty}([0, T])$ with $0 \leq \xi<\varsigma$ on $[0, T]$ and $\xi(0)>a$ and a number $\delta>0$ such that $u_{x} \geq 2 \delta$ a.e. in $B_{T}$, where

$$
B_{T}=\{(x, t): \xi(t) \leq x \leq 1,0 \leq t \leq T\} .
$$

ProOF. By Lemma 3.2 and a straightforward compactness argument there exist a function $\xi \in C^{\infty}([0, T])$ and an $\varepsilon>0$ with $\varepsilon \leq \xi<\varsigma$ on $[0, T]$ such that $u_{x} \geq 0$ a.e. in the set $\{(x, t): \xi(t)-\varepsilon \leq x \leq 1,0 \leq t \leq T\}$. Since $u_{0}^{\prime}>0$ near $\varsigma(0)$ we may assume that $u_{0}^{\prime}>0$ on $[\xi(0), 1]$. By the strong maximum principle, applied to $u_{x}$ in a neighborhood of the curve $x=\xi(t)$, there exists a number $\delta$, with $0<2 \delta \leq f$ on $[0, T]$, such that $u_{x} \geq 2 \delta$ on the parabolic boundary of $B_{T}$. Then Lemma 3.3 follows from the maximum principle for $u_{x}$.

LEMMA 3.4. Let the hypotheses $\mathrm{H} 1-\mathrm{H} 4$ and assumption $\mathrm{A} 2$ be satisfied and let $u_{n}$ be the solution of Problem $\mathrm{I}_{n}$. Then there exists an $N \in \mathbf{Z}^{+}$such that $u_{n x} \geq \delta>0$ on $B_{T}$ for all $n \geq N$, where $\delta$ and $B_{T}$ are given by Lemma 3.3.

ProOF. The approximating functions $u_{0 n}$ and $f_{n}$ in Problem $\mathrm{I}_{n}$ can be chosen in such a way that $u_{0 n}^{\prime} \geq \delta$ on $[\xi(0), 1]$ and $f_{n} \geq \delta$ on $[0, T]$.

Since the curve $x=\xi(t)$ lies entirely in the region where $u$ is a classical solution, it follows from standard a priori estimates [9] that $u_{n} \rightarrow u$ in $C^{2,1}(\overline{\mathcal{N}})$, where 
$\mathcal{N}$ is a neighborhood of $x=\xi(t)$ in $Q_{T}$. Hence it follows from Lemma 3.3 that $u_{n x}(x, t) \geq \delta$ if $x=\xi(t)$ for $n$ large enough.

Thus $u_{n x} \geq \delta$ on the parabolic boundary of $B_{T}$ for $n$ large, and Lemma 3.4 follows form the maximum principle.

4. Theorem 1.1. In this section we shall prove Theorem 1.1. The main step in the proof is to show the uniform boundedness of $u_{n t}$ near $x=\zeta(t)$. At this point we distinguish two cases. In Lemma 4.1 we give a proof in the case that

$$
c(u(1, t))=1 \text { for all } t \in[0, T] .
$$

In Lemma 4.2 we consider the general case, but there we shall need concavity of $c(s)$ near $s=0$.

Below $C$ denotes a generic constant.

LEMMA 4.1. Let hypotheses $\mathrm{H} 1-\mathrm{H} 4$ and assumption $\mathrm{A} 2$ be satisfied, and let $u$ resp. $u_{n}$ be the solution of Problem $\mathrm{I}$ resp. $\mathrm{I}_{n}$, with $u_{0 n}$ defined by Lemma 2.2. If $u$ satisfies (4.1), then there exists a constant $C$ such that, for $n$ large enough, $\left|u_{n t}\right| \leq C$ in $B_{T}$, where $B_{T}$ is defined by (3.6).

PROOF. We extend the solution $u$ by

$$
u(x, t)=f(t)(x-\varsigma(t)), \quad x \geq 1,0 \leq t \leq T .
$$

Since $c(u(1, \cdot))=1$ on $[0, T]$, it follows that $u$ can be considered as the solution of Problem I on $[0, A] \times[0, T]$ for any $A \geq 1$, where the boundary condition $u_{x}=f$ holds at $x=A$ instead of at $x=1$. This argument implies that we may assume without loss of generality that

$$
\varsigma(t)<1 \text { on }[0, T] \text {. }
$$

By Lemma 3.4, uniform boundedness on $B_{T}$ of $u_{n t}$ and of $\eta_{n}$ are equivalent for $n$ large enough. Below we shall estimate $\eta_{n}$.

We observe that $\eta_{n}$ is the classical solution of the initial-boundary value Problem $\mathrm{II}_{n}$ :

$$
\begin{cases}\eta_{t}=\eta_{x x} / c_{n}^{\prime}\left(u_{n}\right)-\left(\eta^{2}\right)_{x}, & \text { in } B_{T}, \\ \eta_{x}=-f_{n}^{\prime} / f_{n}+c_{n}^{\prime}\left(u_{n}\right) \eta^{2}, & x=1,0 \leq t \leq T \\ \eta=-u_{n t} / u_{n x}, & x=\xi(t), 0 \leq t \leq T \\ \eta(\cdot, 0)=-u_{0 n}^{\prime \prime} / c^{\prime}\left(u_{0 n}\right) u_{0 n}^{\prime}, & \xi(0) \leq x \leq 1\end{cases}
$$

Here (4.3) follows from Lemma 3.1, and (4.4) is derived from

$$
\begin{aligned}
& \eta_{n x}=-\left(\frac{u_{n t}}{u_{n x}}\right)_{x}=-\frac{u_{n x t}}{u_{n x}}+\frac{u_{n t} u_{n x x}}{u_{n x}^{2}}=-\frac{f_{n}^{\prime}}{f_{n}}+c_{n}^{\prime}\left(u_{n}\right)\left(\frac{u_{n t}}{u_{n x}}\right)^{2} \\
& \quad \text { for } x=1,0 \leq t \leq T .
\end{aligned}
$$

To prove Lemma 4.1, we construct comparison functions which do not depend on $n$.

Define, for $A>0$,

$$
\underline{\eta}(x, t)=-A(x-1)-e^{2 A(t+1)} \quad \text { on } B_{T} .
$$

We claim that, for $A$ and $n$ large, $\eta$ satisfies (4.3)-(4.6) with equality replaced by $\leq$. Concerning (4.3) and (4.4), this follows from a simple calculation. Concerning (4.5) 
and (4.6) we use the uniform boundedness of $\eta_{n}$ on $x=\xi(t)$, resp. $B_{T} \cap\{t=0\}$, which, at $x=\xi(t)$, follows from the proof of Lemma 3.4, and, at $t=0$, from Lemma 2.2 .

Hence, for $A$ and $n$ large, $\eta$ is a subsolution of Problem $\mathrm{II}_{n}$ and thus, by the maximum principle $[\mathbf{4}]$,

$$
\eta_{n} \geq \underline{\eta} \text { in } B_{T} .
$$

Next we define

$$
\bar{\eta}(x, t)=B(x+1), \quad(x, t) \in B_{T} .
$$

As above, it follows that, for $B$ large enough $\bar{\eta}$ satisfies (4.3), (4.5) and (4.6) with equality replaced by $\geq$. To prove the same for (4.4), we have to show that

$$
c_{n}^{\prime}\left(u_{n}(1, t)\right) \rightarrow 0 \quad \text { as } n \rightarrow \infty
$$

which enables us to neglect the term $c_{n}^{\prime}\left(u_{n}\right) \eta^{2}$ in (4.4). Accepting (4.8) for the moment, it follows that $\bar{\eta}$ is a supersolution of Problem $\mathrm{II}_{n}$ for $B$ and $n$ large, and thus

$$
\eta_{n} \leq \bar{\eta} \quad \text { in } B_{T} .
$$

Lemma 4.1 follows from (4.7) and (4.9).

So it remains to prove (4.8). For every $\phi \in C([0, T])$ with $0 \leq \phi<\zeta$ on $[0, T]$ we known that $u_{n}(\phi(t), t) \rightarrow u(\phi(t), t)$ in $C([0, T])$ as $n \rightarrow \infty$. Since $u(\varsigma(t), t)=0$ and $u_{n x}(\zeta(t), t) \geq \delta>0$ on $B_{T}$, it follows from (4.2) that there exists an $\varepsilon>0$ such that $u_{n}(1, \cdot) \geq \varepsilon$ on $[0, T]$ for $n$ large enough. Since $c_{n}^{\prime} \rightarrow 0$ uniformly on $[\varepsilon, \infty)$ as $n \rightarrow \infty$, we obtain (4.8).

LEMMA 4.2. Let hypotheses $\mathrm{H} 1-\mathrm{H} 4$ and assumptions $\mathrm{A} 1, \mathrm{~A} 2$ be satisfied. Let $u_{n}$ be the solution of Problem $\mathrm{I}_{n}$ with $u_{0 n}$ defined by Lemma 2.2. Then there exists a constant $C$ such that for $n$ large enough $\left|u_{n t}\right| \leq C$ in $B_{T}$.

PROOF. The upper bound for $u_{n t}$ follows, as in the proof of Lemma 4.1, by constructing a lower bound for $\eta_{n}$.

The construction of a supersolution of Problem $\mathrm{II}_{n}$ fails, because we can no longer neglect the term $c_{n}^{\prime}\left(u_{n}\right) \eta^{2}$ in (4.4). For that reason we give a different proof, where we need the concavity of $c(s)$ near $s=0$.

We choose $\varepsilon>0$ and $c_{n}$ such that for all $n c_{n}(s)$ is concave on $(-4 \varepsilon, \infty)$. It follows easily from the uniform continuity of $c(u(1, \cdot))$ on $[0, T]$ that there exists a finite partition

$$
0=t_{0}<t_{1}<\cdots<t_{m}=T
$$

of the interval $[0, T]$ such that for all $i=1, \ldots, m$ either $u(1, t)>-2 \varepsilon$ or $u(1, t)<$ $-\varepsilon$ on $\left(t_{i-1}, t_{i}\right)$.

If $u(1, t)<-\varepsilon$ on $\left(t_{i-1}, t_{i}\right), u$ is a classical solution on $[0,1] \times\left[t_{i-1}, t_{i}\right]$. Thus if $t_{i-1} \neq 0, u_{t}$ and $u_{n t}$ are uniformly bounded on these subsets and, if $t_{i-1}=0$, on compact subsets of $\left\{[0,1] \times\left[0, t_{1}\right]\right\} \backslash\{[0, a] \times\{0\}\}$. In particular, if $m$ in (4.10) is chosen minimal, then

$$
\begin{cases}u_{n t} \geq-C & \text { on }[0,1] \times\left\{t_{i}\right\}, i=1, \ldots, m, \\ u_{n t} \geq-C & \text { on }[a, 1] \times\{0\} .\end{cases}
$$


It remains to prove that, for some $\phi \in C^{\infty}([0, T])$ with $\phi(0)>a$ and $\phi<\varsigma$ on $[0, T]$, and for $n$ large

$$
u_{n t} \geq-C \text { in } \mathcal{K}_{i}=\left\{(x, t): \phi(t) \leq x \leq 1, t_{i-1} \leq t \leq t_{i}\right\}
$$

for all $i$ with $u(1, t)>-2 \varepsilon$ on $\left(t_{i-1}, t_{i}\right)$. We fix such an $i$. We choose $\phi$ such that $u>-3 \varepsilon$ on $K_{i}$. Then, for $n$ large, $u_{n}>-4 \varepsilon$ on $K_{i}$. Defining $q_{n}=u_{n t}$ it follows from the concavity of $c_{n}$ that

$$
c_{n}^{\prime}\left(u_{n}\right) q_{n t}=-c_{n}^{\prime \prime}\left(u_{n}\right) u_{n t}^{2}+q_{n x x} \geq q_{n x x} \text { on } \mathcal{K}_{i} .
$$

In addition, $u_{n t} \geq-C$ on the lateral boundary $x=\phi(t)$ of $\mathcal{K}_{i}$ (where $u$ is a classical solution) and on $K_{i} \cap\left\{t=t_{i-1}\right\}$ (by (4.11)). Finally, $\left(u_{n t}\right)_{x}=f_{n}^{\prime}$ is uniformly bounded on the lateral boundary $x=1$. Hence (4.12) follows from the maximum principle.

ProOF OF THEOREM 1.1. (iii) By Lemmas 4.1 and 4.2 and from the fact that $u$ is a classical solution in $Q_{T} \backslash B_{T}$, it follows that $u_{n}$ is uniformly Lipschitz continuous with respect to $t$ (and also, by Proposition 2.1(i), with respect to $x$ ) on $[0,1] \times[\tau, T]$ for any $\tau>0$. Hence the limit function $u$ is Lipschitz continuous on $[0,1] \times[\tau, T]$, and $u_{t} \in L_{\mathrm{loc}}^{\infty}([0,1] \times(0, T])$.

(iv) Since, by $(2.1),(c(u))_{t}=u_{x x}=0$ if $x>\varsigma(t)$, and since $(c(u))_{t}=u_{x x} \in$ $C(D)$, it is enough to prove that

$$
\lim _{\substack{(x, t) \rightarrow\left(\zeta\left(t_{0}\right), t_{0}\right) \\(x, t) \in \mathcal{D}}}(c(u(x, t)))_{t}=0 \quad \text { if } u\left(\varsigma\left(t_{0}\right), t_{0}\right)=0 .
$$

This follows at once from (iii) and the fact that $c^{\prime}\left(0^{-}\right)=0$.

(i) It is sufficient to prove that $\zeta(t)$ is uniformly Lipschitz continuous on $\{t \in$ $[0, T]: \varsigma(t)<1\}$.

We fix $\tau \in[0, T]$ with $\varsigma(\tau)<1$. Then $\varsigma(t)<1$ in a neighborhood $O_{\tau}$ of $\tau$. By Lemma 3.3 the level curves $x=\zeta_{\varepsilon}(t)$ with $u\left(\zeta_{\varepsilon}(t), t\right)=-\varepsilon$ are well defined smooth curves for $\varepsilon$ small enough and $t \in O_{\tau}$. Since $\zeta_{\varepsilon} \nearrow \zeta$ on $O_{\tau}$ as $\varepsilon \searrow 0$ it is sufficient to prove that

$$
\left|\zeta_{\varepsilon}^{\prime}\right| \leq C \text { on } O_{\tau}
$$

where $C$ does not depend on $\varepsilon$ and $\tau$.

We derive from the fact that $(d / d t) u\left(\zeta_{\varepsilon}(t), t\right)=0$ on $O_{\tau}$, that

$$
\zeta_{\varepsilon}^{\prime}(t)=-u_{t}\left(\zeta_{\varepsilon}(t), t\right) / u_{x}\left(\zeta_{\varepsilon}(t), t\right), \quad t \in O_{\tau} .
$$

Since, for $\varepsilon$ small, $u_{x}$ is bounded away from zero (by Lemma 3.3), (4.13) follows from the fact that $u_{t}$ is bounded in a neighborhood in $\overline{Q_{T}}$ of $x=\varsigma(t)$.

(ii) Because, by (2.1), $u_{x}=f$ if $x>\varsigma(t)$ and since $u_{x} \in C(D)$, it is enough to show that

$$
\lim _{\substack{(x, t) \rightarrow\left(s\left(t_{0}\right), t_{0}\right) \\(x, t) \in \mathcal{D}}} u_{x}(x, t)=f\left(t_{0}\right), \quad 0 \leq t_{0} \leq T .
$$

Since $\varsigma \in C([0, T])$, we only have to show that

$$
\lim _{\varepsilon \searrow 0} u_{x}(\zeta(t)-\varepsilon, t)=f(t) \quad \text { uniformly on }[0, T] \text {. }
$$

Because $u_{x x}$ is bounded near $x=\zeta(t), u_{x}(\zeta(t)-\varepsilon, t)$ converges uniformly on $[0, T]$ to a continuous function $g(t)$ on $[0, T]$. By Proposition 2.1(i), $u(\cdot, t) \in C^{1}([0,1])$ for a.e. $t \in[0, T]$, and hence $f(t)=g(t)$ a.e. on $[0, T]$. Since both $f$ and $g$ are continuous, $f \equiv g$ on $[0, T]$. 
5. Theorem 1.2. We proceed as in the proof of Theorem 1.1: first we use Problem $\mathrm{II}_{n}$ to obtain estimates for $\eta_{n}$.

LEMMA 5.1. Let the assumptions of Theorem 1.2 be satisfied, let $u_{0 n}$ be defined by Lemma 3.2, and let $f_{n}$ satisfy the compatibility condition

$$
\left(u_{0 n}^{\prime \prime}(1) / c_{n}^{\prime}\left(u_{0 n}(1)\right)\right)^{\prime}=f_{n}^{\prime}(0) .
$$

Let $\eta_{n}$ be defined by (3.2). Then $\left|\eta_{n x}\right| \leq C$ on $B_{T}$ for $n$ large enough.

PROOF. First we observe that $\eta_{n x}$ is uniformly bounded on the parabolic boundary of $B_{T}$. On the lateral boundary $x=\xi(t)$ this follows from the fact that $u$ is a classical solution of Problem I and $u_{n x} \rightarrow u_{x}$ in $C^{2,1}$ near $x=\xi(t)$. On $B_{T} \cap\{t=0\}$ it follows from (2.5), where we use that

$$
\eta_{n x}=-\frac{u_{n x t}}{u_{n x}}+\frac{u_{n t} u_{n x x}}{\left(u_{n x}\right)^{2}}=-\frac{1}{u_{n x}}\left(\frac{u_{n x x}}{c_{n}^{\prime}\left(u_{n}\right)}\right)_{x}+c_{n}^{\prime}\left(u_{n}\right) \eta_{n} .
$$

Finally, (4.4) implies the uniform boundedness of $\eta_{n x}$ on the lateral boundary $x=1$. Here we use the uniform bound on $\eta_{n}$, established in $\S 4$.

Differentiating equation (4.3) with respect to $x$, we find that $w=\eta_{n x}$ satisfies

$$
w_{t}=\left\{w_{x} / c_{n}^{\prime}\left(u_{n}\right)-2 \eta_{n} w\right\}_{x} .
$$

By $\left[9\right.$, p. 181, Theorem 7.1], this implies that $\eta_{n x}$ is uniformly bounded on $B_{T}$.

PROOF OF THEOREM 1.2. (ii) By Lemma 5.1 and (5.1), $u_{n x t}$ is uniformly bounded in $B_{T}$, and thus also in $[0,1] \times[\tau, T]$ for all $\tau \in(0, T)$. In view of Theorem 1.1(iii), $u_{n x x}$ is uniformly bounded in $[0,1] \times[\tau, T]$ and hence $u_{n x}$ is Lipschitz continuous in this set. Since the Lipschitz constant does not depend on $n$, the same is true for $u_{x}$, which shows that $u_{x t} \in L_{\text {loc }}^{\infty}([0,1] \times(0, T])$.

(i) + (iii) Using that $u_{x t}$ is bounded, it follows that $u_{t}(\zeta(t)-\varepsilon, t)$ converges uniformly on $[0, T]$ as $\varepsilon \searrow 0$. Hence $u_{t} \in C\left(\bar{D} \cap Q_{T}\right)$.

Let $O_{\tau}$ and $\zeta_{\varepsilon}(t)$ be defined as in the proof of Theorem 1.1(i). Then, for $\varepsilon$ small,

$$
\zeta_{\varepsilon}^{\prime}(t)=-u_{t}\left(\zeta_{\varepsilon}(t), t\right) / u_{x}\left(\zeta_{\varepsilon}(t), t\right), \quad t \in O_{\tau} .
$$

Since $\left(u_{t} / u_{x}\right)_{x}$ is bounded, and $\zeta \in C([0, T])$, it follows that $\zeta_{\varepsilon} \rightarrow \zeta$ in $C^{1}\left(\bar{O}_{\tau}\right)$ and $\zeta^{\prime}$ is given by (1.8).

(iv) By (2.1)

$$
u_{t}=f^{\prime}(x-\varsigma)-f \zeta^{\prime}, \quad \zeta(t)<x<1 .
$$

Hence $u_{t} \in C\left(Q_{T} \backslash D\right)$. In view of (ii), $u_{t} \in C\left(Q_{T} \cap \bar{D}\right)$. Finally, by (1.8) and (5.2), $u_{t}$ is continuous across the interface $x=\varsigma(t)$.

6. A Dirichlet boundary condition. In this section we briefly indicate how the main results of this paper can be generalized to the case that the Neumann condition at $x=1$ is replaced by a Dirichlet condition:

$$
u(1, t)=g(t)>0 \text { on }[0, T],
$$

where $g$ is a Lipschitz continuous and positive function on $[0, T]$. We shall, for convenience, refer to this problem as the Dirichlet problem, although we still have $u_{x}=0$ at $x=0$. Again (see [8]) there exists a unique weak solution $u$ and, as long as the medium is not completely saturated, i.e.,

$$
c(u(\cdot, t)) \not \equiv 1 \text { on }[0,1] \text { for all } 0 \leq t \leq T,
$$


the interface $x=\varsigma(t)$ is continuous and satisfies

$$
0<\varsigma<1 \text { on }[0, T] \text {. }
$$

We claim that in the situation described above, Theorems 1.1 and 1.2 still hold, if in assumption A2 the condition $u_{0}^{\prime}(\zeta(0))=f(0)$ is replaced by

$$
u_{0}^{\prime}(\zeta(0))=g(0) /(1-\varsigma(0)) \text {. }
$$

Observe that, in view of $(6.2), c(u(1, \cdot)) \equiv 1$ on $[0, T]$ and hence assumption A1 can be omitted. Of course we do not need the assumptions concerning $f(t)$ anymore.

To prove our claim, we notice that in the saturated region $u_{x x}=0$ a.e. and thus we may assume that (cf. (2.1))

$$
u(x, t)=\frac{g(t)}{1-\varsigma(t)}\{x-\varsigma(t)\}, \quad x \leq \varsigma(t) \leq 1,0 \leq t \leq T .
$$

In particular $u$ can be considered as the solution of Problem I with the Neumann condition $u_{x}(1, t)=f(t)$, where

$$
f(t)=g(t) /(1-\varsigma(t))
$$

Since $\zeta \in C([0, T])$ and $\zeta<1$ on $[0, T], f$ is a positive and continuous function, but not, as was assumed in the previous sections, a Lipschitz continuous function. In the first step of the proof of Theorem 1.1, namely Lemma 3.3, we did not use the Lipschitz continuity of $f$, and so Lemma 3.3 still holds.

In the rest of the proof of Theorem 1.1 we consider $u$ as the solution of the Dirichlet problem. In particular, $u$ can be obtained as the limit of smooth solutions $u_{n}$ of an approximating Dirichlet problem, with $g$ replaced by $g_{n}$. The main line of the proof of Theorem 1.1 remains the same. Only at two parts, which we discuss below, the proof has to be adapted.

First, to prove Lemma 3.4, we need to know that $u_{n x}(1, \cdot)$ is positive and bounded away from 0 on $[0, T]$ for $n$ large. To do this, a supersolution $\bar{u}_{n}(x, t)$ with the properties $\bar{u}_{n x}(1, \cdot) \geq \delta>0$ and $\bar{u}_{n}(1, \cdot)=g_{n}$ on $[0, T], \bar{u}_{n} \geq 0$ in $Q_{T}$, can be used as a barrier function. The construction of $\bar{u}_{n}$ is left to the reader.

Secondly, the proof of Lemma 4.1 is simplified since the boundary condition (4.4) for $\eta_{n}$ at $x \doteq 1$ is replaced by

$$
\eta_{n}(1, t)=-g_{n}^{\prime}(t) / u_{n x}(1, t), \quad 0 \leq t \leq T .
$$

Hence $\eta_{n}$ is uniformly bounded on the boundary $x=1$ and the construction of a subsolution and a supersolution is straightforward.

Finally we observe that, once we have proved Theorem 1.1, we know that $\zeta$ is Lipschitz continuous, and so is $f$, given by (6.3). Hence Theorem 1.2 follows at once if we consider $u$ as the solution of the Neumann problem.

\section{REFERENCES}

1. W. H. Alt and S. Luckhaus, Quasilinear elliptic-parabolic differential equations, Math. Z. 183 (1983), 311-341.

2. M. Bertsch, M. E. Gurtin and D. Hilhorst, On a degenerate diffusion equation of the form $c(z)_{t}=\phi\left(z_{x}\right)_{x}$ with application to population dynamics, J. Differential Equations (to appear). 
3. E. DiBenedetto and R. Gariepy, Local behaviour of solutions of an elliptic-parabolic equation (to appear).

4. A. Friedman, Partial differential equations of parabolic type, Prentice-Hall, Englewood Cliffs, N.J., 1964.

5. M. E. Gurtin, R. C. MacCamy and E. A. Socolovsky, A coordinate transformation for the porous media equation that renders the free-boundary stationary, Quart. Appl. Math. 42 (1984), 345-357.

6. J. Hulshof, An elliptic-parabolic free boundary problem: continuity of the interface, Math. Inst. Univ. Leiden, Report No. 10, 1985.

7. ary, Math. Inst. Univ. Leiden, Report No. 21, 1985.

8. J. Hulshof and L. A. Peletier, An elliptic-parabolic free boundary problem, Math. Inst. Univ. Leiden, Report No. 14, 1984; Nonlinear Anal. (to appear).

9. O. A. Ladyzenskaja, V. A. Solonnikov and N. N. Ural'ceva, Linear and quasilinear equations of parabolic type, Transl. Math. Monographs, vol. 23, Amer. Math. Soc., Providence, R.I., 1968.

MATHEMATICAL INStitute, UNiversity OF LEIDEN, LEIDEN, THE NETHERLANDS 